\title{
DE QUELQUES TRAITS PRÉHILALIENS DANS LE PARLER DES OULED BOUMALEK / BOUHOUDA (TAOUNATE)
}

\author{
Rida CHALFI* \\ Faculté Polydisciplinaire d'Errachidia
}

BIBLID [1133-8571] 26 (2019) 05.1-14.

Résumé : L'objectif primordial de ce travail est une brève présentation de quelques caractéristiques linguistiques du parler jebli des Ouled Boumalek, un douar bien enclavé situé à $18 \mathrm{Km}$ du centre Bouhouda, province de Taounate. Dans ce papier, les résultats présentés sont le labeur de quelques enquêtes de terrain auprès d'informateurs natifs, qui ne quittent que rarement la zone de leurs ancêtres, et surtout les femmes âgées, gardiennes du patrimoine culturel par excellence. Pour ce faire, nous avons exploité plusieurs techniques de terrain (observation participante, entretien et questionnaire...). Par cette étude, nous avons mis l'accent sur les différents aspects linguistiques d'un parler préhilalien appartenant à la zone des Jbala (particularités phonétiques / phonologiques / morphologiques et lexicales).

Mots-clés : Parlers préhilaliens, Linguistique, Jbala / Taounate.

\begin{abstract}
Some traits of Ouled Boumalek’s variety in pre-hilalian era: Bouhouda - Taounate». The main objective of this research is to briefly trace some linguistic features of the jebli variety of Ouled Boumalek, a well-established douar located $18 \mathrm{~km}$ far from Bouhouda center in Taounate province. In this paper, the data are the result of a few field surveys with native informants, who rarely leave the area of their ancestors, especially older women as custodians of cultural heritage par excellence. To do this, we exploited several field techniques (engaging listening, interview and questionnaire ....). Through this study, we have focused on the different linguistic aspects of a prehilalian speech belonging to the Jbala zone (phonetic / phonological / morphological and lexical peculiarities).
\end{abstract}

Keywords: Pre-Hilalian Varieties, Linguistics, Jbala, Taounate.

E-mail: ridachalfi@hotmail.com. 


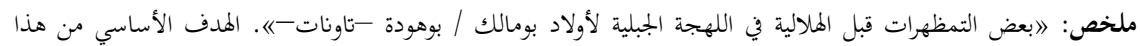

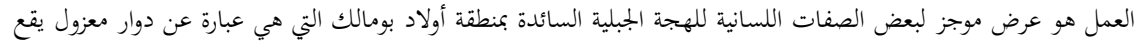

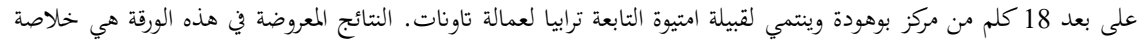

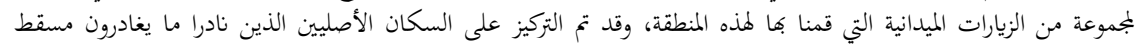

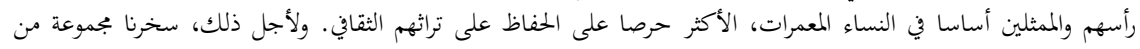

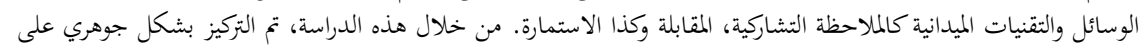

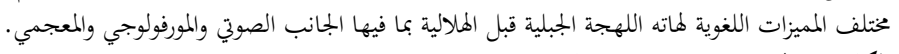

\section{Introduction}

$$
\text { الكلمات المفاتيح: اللهجات فبل الهلالية، اللسانيات، جبالة، تاونات. }
$$

Dans cet article, nous tenterons de présenter brièvement les principaux traits linguistiques caractérisant le parler jebli du douar Ouled Boumalek, une région enclavée située à une trentaine de kilomètres de la ville de Taounate vers le Nord-Ouest et qui appartient à la commune rurale Bouhouda, tribu de Mtioua. Les données exploitées dans cette étude sont le résultat de quelques enquêtes de terrain effectuées entre les mois mars et novembre 2018. Les informations nécessaires à cette investigation sont collectées de manière naturelle auprès d'informateurs natifs, qui ne quittent que rarement la zone de leurs ancêtres, et surtout les femmes âgées, gardiennes du patrimoine culturel par excellence.

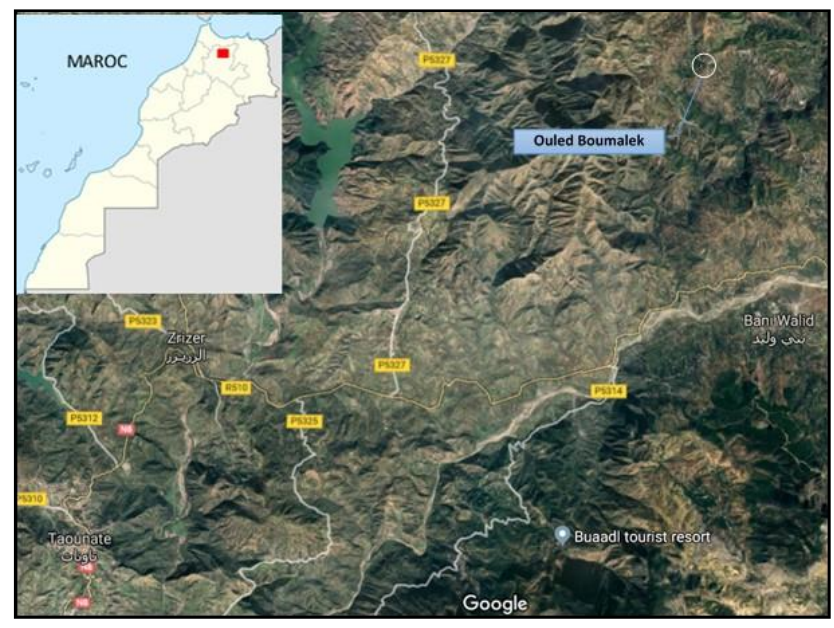

Figure 1 : Carte de situation de la zone d'étude 


\section{Phonétique/phonologie}

\subsection{Coup de glotte}

Dans le parler jebli des Ouled Boumalek (Dorénavant P.J.O.B.), le coup de glotte [?] est l'un des traits les plus frappants. Il est généralement utilisé pour remplacer l'uvulaire occlusive sourde [q].

mša yaliut z-zaytūn $m \uparrow a$ babāh «il a accompagné son père pour cueillir les olives ».

\subsection{Spirantisme}

Le parler d'étude présente également quelques segments spirants [t] [đ] [Đ] [트]

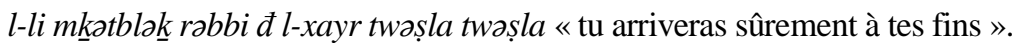

hađa ma kīan «c'est tout ce qu'il y a...»

l-lā yla?ik biha biĐa « que Dieu te protège »

$m \check{S} a \underline{t}$ traggab Sla babaha $m r i ̄ Đ$ « elle a rendu visite à son père malade »

N.B. Il importe de souligner que les désinences du féminin singulier des verbes à l'accompli se forment par l'adjonction du segment [t] .

\subsection{Diphtongues}

L'usage des diphtongues est très fréquent dans notre parler d'étude qui présente deux types : [ay] / [aw].

ntina $w$ jawdek xudam aw xliham « comme tu le veux. Tu peux les prendre ou les laisser ».

\subsection{Quelques traits phonologiques}

L'analyse des données recueillies nous a permis de repérer quelques traits phonologiques comme la métathèse, l'aphérèse, l'apocope et la substitution.

\subsubsection{Métathèse}

\begin{tabular}{|c|l|l|l|}
\hline $\mathrm{C} / \mathrm{C}$ & $\begin{array}{c}\text { Autres parlers } \\
\text { marocains }\end{array}$ & \multicolumn{1}{|c|}{ P.J.O.B. } & Sens français \\
\hline $\mathrm{t} \mathrm{n} \leftrightarrow$ & {$[\mathrm{tənkəl}]$} & {$[$ nətkəl] } & Manger \\
\hline $\mathrm{t} \mathrm{f} \leftrightarrow$ & {$[\mathrm{tfakŠ}]$} & {$[\mathrm{ftakS}]$} & Se séparer \\
\hline $\mathrm{n} \mathrm{d} \leftrightarrow$ & {$[$ maSədnus] } & {$[$ maSəndlus] } & Coriandre \\
\hline
\end{tabular}




\begin{tabular}{|c|c|c|c|}
\hline Š f $\leftrightarrow$ & [1-xurŠif] & [1-XurfiŠ $]$ & Cardon \\
\hline $\operatorname{tr} \leftrightarrow$ & [tərҮəd] & [rətSəd] & Trembler \\
\hline $\mathrm{th} \leftrightarrow$ & [tŠəhha] & [Šətha] & Avoir envie \\
\hline
\end{tabular}

\subsubsection{Aphérèse}

\begin{tabular}{|c|c|c|}
\hline Autres parlers & P.J.O.B. & S.F. \\
\hline [Sṭitu] & [Øtititu] & Je lui ai donné \\
\hline [maŠi hada wəqtha] & [muŠay Øda wa?ta] & Ce n'est pas le bon moment \\
\hline [wahad l-mra] & [Øha 1-mra] & Une femme \\
\hline [la tədrəbŠ xuk] & [la ØĐrabŠi xāk] ] & Ne frappe pas ton frère! \\
\hline [ma təmŠsiš mৎāh] & [la ØmŠiši mSāh] & Ne pars pas avec lui! \\
\hline
\end{tabular}

Remarque : les phonèmes supprimés sont marqués par le symbole $\emptyset$. Il est à noter que, dans le P.J.O.B., les verbes conjugués à l'impératif perdent leurs préfixes dans le cas où ils sont précédés par le morphème de négation "la" (les deux derniers exemples).

\subsubsection{Apocope}

\begin{tabular}{|c|c|c|}
\hline Autres parlers & P.J.O.B. & S.F. \\
\hline [tŠiša] & [ť̌siš & Semoule \\
\hline [həyyəd mən hadaya ] & [ḥəyyØ mØ hadāyØ ] & Casse-toi ! \\
\hline [layn mŠa] & [layØ mŠa] & Où est-ce qu'il est parti ? \\
\hline [waḥad 1-muŠkil] & [haØ 1- muŠkil] & Un problème... \\
\hline [Škun ža] & [ŠkuØ ža] & Qui est venu? \\
\hline [fayn mŠìt] & [fayØ mŠìt $\underline{t}$ & Où est-ce que tu es parti? \\
\hline [Sad wṣəlt mən ș-ṣuq] & [؟aØ wșəlt mØ ș-șu?] & Je viens d'arriver du marché \\
\hline [qiș had Š-Ši] & [qiș haØ Š-Ši] & Goûte ceci ! \\
\hline [sədd 1-bāb] & [suØ l-bāb] & Ferme la porte! \\
\hline
\end{tabular}




\subsubsection{Substitution}

\begin{tabular}{|c|c|c|c|}
\hline $\begin{array}{c}\text { Les } \\
\text { phonèmes }\end{array}$ & $\begin{array}{l}\text { Autres } \\
\text { parlers }\end{array}$ & P.J.O.B. & S.F. \\
\hline $\mathrm{m} \mathrm{z} \rightarrow$ & [zormumiya] & [zorzumiya] & Lézard \\
\hline $\mathrm{yg} \rightarrow$ & [yabəs] & [gabəs] & Sec \\
\hline $\mathrm{d} \underline{\mathrm{t}} \rightarrow$ & [buhdi] & [bahti & Moi seul \\
\hline $\ln \rightarrow$ & [tzəlzəl] & [tzənzəl] & Glisser \\
\hline $\mathrm{d} l \rightarrow$ & [ž-žəgidid] & [ǰagdil] & Trop salé \\
\hline $\mathrm{dg} \rightarrow$ & [dwāz] & [gwāz] & Repas à base de légumineuses \\
\hline $\mathrm{dg} \rightarrow$ & [dāz] & [gāz] & Il est passé \\
\hline $\mathrm{kg} \rightarrow$ & [s-sāk] & [s-sāg] & $\mathrm{Sac}$ \\
\hline $\mathrm{bf} \rightarrow$ & [bhāall] & [fhạa] & Comme \\
\hline $\mathrm{q} x \rightarrow$ & [nəqqəș] & [nəxxəș] & Baisser \\
\hline$d \underline{t} \rightarrow$ & [rəmmayḍa] & [rummayța] & Cache-cahe \\
\hline $\mathrm{ts} \rightarrow$ & [mətqadda] & [məs?adda] & Bien présentée \\
\hline
\end{tabular}

On trouve également le phénomène de substitution dans de nombreux termes empruntés au français comme :

\begin{tabular}{|l|l|l|l|}
\hline Les phonèmes & \multicolumn{1}{|c|}{ Autres parlers } & \multicolumn{1}{c|}{ P.J.O.B. } & \multicolumn{1}{c|}{ S.F. } \\
\hline 1-------r & [tilifūn] & [tirifūn] & Téléphone \\
\hline 1-------r & [təlfaza] & [tərfaza] & Télévision \\
\hline 1-------r & [țələkumund] & [tərəkumund] & Télécommande \\
\hline l-------r & [țunubīl] & [țunubīr] & Automobile \\
\hline v------b & [vitrina] & [bitrina] & Vitrine \\
\hline
\end{tabular}

\section{Morphologie}

\subsection{Genre}

Certains noms féminins dans d'autres parlers marocains (Fès, Kénitra...) sont considérés masculins dans notre parler d'étude. À titre d'exemple, nous signalons le cas de noms ržəl «pied» et yadd «main» qui sont traités masculins dans le P.J.O.B.

a) rəžli mritt «pied à moi malade », « mon pied est malade »

b) yədda fih l-hinna « main elle dedans henné », « elle a mis du henné sur sa main ». 


\subsection{Duel}

Dans le parler en question, le duel se forme par la suffixation du morphème -ayzn aux substantifs.

\begin{tabular}{|l|l|}
\hline \multicolumn{1}{|c|}{ Singulier } & \multicolumn{1}{c|}{ Duel } \\
\hline$[$ mudd] unité de mesure & [muddayən] deux unités de mesure $(33 \mathrm{~kg})$ \\
\hline$[$ mərra $]$ une fois & [mərtayən] deux fois \\
\hline$[$ saSa] une heure & [saStayən] deux heures \\
\hline$[$ lila $]$ une nuit & {$[$ liltayən] deux nuits } \\
\hline
\end{tabular}

\subsubsection{Mots renvoyant aux parties du corps}

\begin{tabular}{|l|l|}
\hline \multicolumn{1}{|c|}{ Singulier } & \multicolumn{1}{c|}{ Duel } \\
\hline$[$ tđa] sein & [təđđayən] deux seins \\
\hline$[$ yədd] main & [yəddayən] deux mains \\
\hline$[$ rjəl] pied & [rəjlayən] deux pieds \\
\hline$[$ Yayn] œil & [Yaynayən] deux yeux \\
\hline$[$ wđən] oreille & [wəđnayən] deux oreilles \\
\hline$[$ xədd] joue & [xəddayən] deux joues \\
\hline
\end{tabular}

De plus, nous pouvons compter deux objets par le biais du nom de nombre žawž « deux » suivi du mot au pluriel.

\begin{tabular}{|c|c|}
\hline Singulier & Duel \\
\hline [laymuna] orange & [žawž laymunāt] deux oranges \\
\hline [dār] maison & [žawž dyār] deux maisons \\
\hline [?dib] bâton & [žawž Piḍbān] deux bâtons \\
\hline [luSma] bouchée & [žawž luSmāt] deux bouchées \\
\hline [dawra] tournée & [žawž dawrāt] deux tournées \\
\hline
\end{tabular}

Remarque : en duel, la quasi-majorité des mots se construit par le moyen de $\check{z} a w z ̌$. Duel $=\check{z} a w \check{z}+$ pluriel du nom. 


\subsection{Pluriel}

Certains substantifs qui se terminent par $-i$ forment leur pluriel par la suffixation de -ìn. Ci-après, nous présenterons deux cas de figures, l-ma / l-fül.

a) Le substantif $l-m a \ll$ eau $»$ :

l-ma bardīn «l'eau est froide »

l-ma sxixnin «l'eau est chaude »

l-ma dafyin « l'eau est tiède »

l-ma hamyīn «l'eau est brûlante »

À l'instar de la langue amazighe, les locuteurs des Ouled Boumalek utilisent le substantif $l$-ma au pluriel ${ }^{(1)}$ au lieu du singulier, ce qui corrobore l'idée du substrat amazighe.

b) Le substantif $l$-fūl «fèves». À l'opposé des autres parlers où le substantif $l$-fül est utilisé au singulier, le même substantif est utilisé toujours au pluriel dans le P.J.O. B. nous citons quelques exemples :

\begin{tabular}{|c|c|}
\hline Autres parlers & P.J.O.B. \\
\hline [l-fül țayab] fèves cuites & [l-fül țaybīn] fèves cuites \\
\hline [l-fül mzəSlək] fèves écrasées & [l-fūl mzaSlkīn] fèves écrasées \\
\hline$[l$-fül mŠarmal] fèves épicées & [l-fūl mŠarmlīn] fèves épicées \\
\hline [l-fül $m f \partial w w ə r]$ fèves cuites à la vapeur & [l-fül mfawrīn] fèves cuites à la vapeur \\
\hline
\end{tabular}

\subsection{Diminutif}

Dans notre zone d'étude, nous avons constaté que l'usage des diminutifs par les sujets enquêtés est très fréquent, surtout chez les femmes âgées et les enfants. Les formes diminutives collectées sont assez variées et aucune règle ne régit leur formation. Exemples :

محمد شفيق' لمحة عن ثلاثة وثلاثين قرنا من تاريخ الامازيغيين (1) 


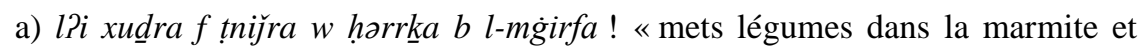
tourne elle avec la cuillère de bois ! », «tu mets les légumes dans la marmite et tu fais tourner le tout avec la cuillère en bois !».

b) ha l-mimāt sxixnīn ma ndiram $f$ n-nibira «cette eau chaude vais la mettre dans le réfrigérateur », « je mettrai cette eau chaude dans le réfrigérateur ».

\subsection{Pronoms}

\subsubsection{Pronoms personnels}

Dans le parler en question, nous avons relevé des pronoms personnels indépendants et des pronoms susceptibles d'être suffixés à des verbes et à des substantifs.

\subsubsection{Pronoms personnels isolés}

\begin{tabular}{|l|l|}
\hline \multicolumn{1}{|c|}{ Singulier } & \multicolumn{1}{c|}{ Pluriel } \\
\hline 1.c. [?ana] moi & 1.c. [hna] nous \\
\hline 2.c. [nta] toi & 2.c. [ntuma] [ntum] vous \\
\hline 3.m. [huwwa] lui & 3.c. [huma] ils / elles \\
\hline 3.f. [hiyya] elle & \\
\hline
\end{tabular}

Remarque : excepté la troisième personne du singulier qui marque le masculin et le féminin, les autres pronoms ne distinguent pas le genre.

2.5.1.2. Pronoms personnels suffixés

2.5.1.2.1. Les pronoms suffixés à un verbe

\begin{tabular}{|l|l|}
\hline \multicolumn{1}{|c|}{ Singulier } & \multicolumn{1}{c|}{ Pluriel } \\
\hline 1.c. $-n \mathrm{i}$ & 1.c. $-n a$ \\
\hline 2.c. $-\partial \underline{k}$ & 2.c. $-\underline{k} u m$ \\
\hline 3.m. $-u$ & 3.c. $-\partial m$ \\
\hline 3.f. $-a$ & \\
\hline
\end{tabular}

Remarque : nous soulignons la disparition du [h], après une consonne, dans les pronoms suffixés de la troisième personne du singulier et du pluriel. 
Exemples :

a) Šuft-Øa b hin?a-đalïb mYa xaha « tout à l'heure, je l'ai vue jouer avec son frère ».

b) wașṣit- $\emptyset^{(2)} \partial m$ yražS-u biǩri « je leur avais demandé de revenir tôt ».

\subsection{Les pronoms suffixés à un nom / le possessif}

Lorsqu'on suffixe un pronom suffixé à un nom, on exprime le possessif.

\begin{tabular}{|l|l|}
\hline \multicolumn{1}{|c|}{ Singulier } & \multicolumn{1}{c|}{ Pluriel } \\
\hline 1.c. $-i$ & 1.c. $-n a$ \\
\hline 2.c. $-\partial \underline{k} /-\bar{a} \underline{k} \underline{k}$ & 2.c. $-\underline{k} u m$ \\
\hline 3.m. $-u$ & 3.c. $-\partial m /-h \partial m$ \\
\hline 3.f. $-a /-h a$ & \\
\hline
\end{tabular}

Le P.J.O.B. ne distingue pas le genre au niveau de la deuxième personne du singulier et fait disparaître le $h$ à la troisième personne du singulier féminin et du pluriel masculin et féminin pour certains items.

Exemples :

a) jat Səmt-əm ha đaba « leur tante paternelle vient d'arriver »

b) baba-həm Pa-ytawmor f l-Girssa « leur père travaille péniblement dans le pré »

c) $b a b-\bar{a} \underline{k}$ Pa-ylag-i $l$-əok « ton père t'appelle »

d) yədd-i $2 a-y \partial h r a ?$ ? $-n i b \breve{S}$ ? $a$ « ma main me fait mal à cause du pénible travail »

Remarque : dans notre zone d'enquête, nous avons constaté que les informateurs utilisent surtout la suffixation pour les noms de parenté et des parties du corps.

Pour le reste des items, la possession est exprimée par la particule dyal qui suit les mots utilisés et subit les transformations possibles selon la personne. Nous citons quelques exemples :

a) $l$-Girșa dyal-i «mon pré »

b) l-ba?ra dyal-a «sa vache »

(2) Dans les exemples, nous avons remplacé le $h$ par $\varnothing$ pour marquer sa disparition. 
c) l-məhrāt dyal-əm « leur araire »

\subsubsection{Les pronoms relatifs}

Le parler des Ouled Boumalek se caractérise par l'utilisation de deux pronoms relatifs qui sont : šay et $l l i$. Ces derniers sont invariables avec le genre et le nombre.

\subsubsection{Le pronom relatif šāy}

a) šây d-dawwar Ylih taliah fhanut «tout ce que tu cherches, tu le trouves dans la boutique »

b) šây Šra $m s$-su? bozzāof «ce qu'il a acheté du souk est vraiment trop »

c) šāy gaz Slina ma gaz Sla hadd «ce qui nous est arrivé, n'est arrivé à personne»

\subsubsection{Le pronom relatif $l l i$}

a) lli dar?ak b xit dar?u b hit ! «oublie celui qui t'oublie !»

b) ha r-raǰil lli saxșa fik «c'est l'homme qui t'a cherché »

\subsubsection{Les pronoms interrogatifs}

Dans notre corpus, nous avons relevé quelques pronoms interrogatifs. Généralement, ces pronoms simples ou composés sont placés en tête des phrases interrogatives. Les plus utilisés par nos locuteurs sont surtout :

2.5.3.1. Les pronoms interrogatifs : šni / šonni / ̌s-

a) šni ?utt ? «qu'as-tu dit?»

b) šni nti mSa dik s-șhiha ? « comment vas-tu ? »

c) š-ma nșawab đaba? «que fais-je maintenant ? »

d) šənni Sməlt f Š́ugl-əzk ? «qu'as-tu fait dans ton travail ? »

\subsubsection{Les pronoms interrogatifs : šk $\underline{\text { L }}$ / škun}

Marçais $^{(3)}$ précise que l'étymologie de šǩ / šǩūn serait Payyu šayßin yakun : quelque chose.

a) šku ntin ? «qui es-tu ?»

b) šǩun lli ja ? «qui c'est qui est venu? »

(3) $1977: 200$. 
c) đi-mən ha tfal? « qui sont les parents de ce garçon ?»

d) $l i$-mən ?a-đlag- $i$ ? «tu appelles qui ? »

\section{Lexique}

Les données collectées auprès des informateurs nous ont permis de mettre en relief la grande richesse lexicale caractérisant le parler des Ouled Boumalek. $\mathrm{Ci}$-après, nous présenterons quelques items appartenant aux différents domaines de la vie rustique de ces habitants.

\begin{tabular}{|c|c|}
\hline P.J.O.B. & Traduction et / ou contexte \\
\hline$[l-b \partial s ̌ b i s ̌ a](\mathrm{N})$. & $\begin{array}{l}\text { - Porter la guigne, le mauvais sort à quelqu'un en } \\
\text { produisant des paroles, un vœu de mauvais augure. }\end{array}$ \\
\hline$[l-? a l l a b a](\mathrm{N})$. & $\begin{array}{l}\text { - Piège traditionnel pour attraper les oiseaux et les } \\
\text { animaux } \\
\text { - [šsdd žawž tyūr } b l \text {-Pallaba }] \\
\text { - Il a attrapé deux oiseaux avec le piège }\end{array}$ \\
\hline [?ajđəm] (N.) & $\begin{array}{l}\text { - Estrade devant la porte de la maison ou parfois à } \\
\text { l'intérieur pour s'asseoir }\end{array}$ \\
\hline$[\text { ?ajmaz }]^{(4)}(\mathrm{N})$. & - Le pouce \\
\hline [l-farnūi?] (N.) & - Bâton sous forme de Y \\
\hline [șottaha] (N.) & - Galet ovoïde et lisse qu'on trouve dans les rivières. \\
\hline $\begin{array}{l}{[\text { balgəm] (N.) }} \\
\text { Plur. [blagim] }\end{array}$ & - Crevasses des pieds et du talon \\
\hline$[$ đayđbān] (N.) & $\begin{array}{l}\text { - Sorte de roseau, corde ou toute chose permettant aux } \\
\text { branches de la vigne de grimper. }\end{array}$ \\
\hline [?ayənyānn] (N.) & $\begin{array}{l}\text { - Trois grosses pierres formant un réchaud en terre } \\
\text { [kanūn] }\end{array}$ \\
\hline $\begin{array}{l}\text { [đ-đəyyāba] (N.) } \\
\text { Plur. [đwayəb] }\end{array}$ & - Marmite fabriquée en terre \\
\hline [labī? $]$ (N.) & - Dépannage \\
\hline 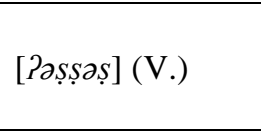 & $\begin{array}{l}\text { - Couper en petits morceaux } \\
\text { - [Pașşș̣ } I-\{w a \bar{a}]] \\
\text { - Il a coupé le bois. }\end{array}$ \\
\hline
\end{tabular}

(4) Dans la région des Ouled Azam, le mot est prononcé [Pagməz] 


\begin{tabular}{|c|c|}
\hline$[t \partial r \underline{r} \partial s](\mathrm{V})$. & $\begin{array}{l}\text { - Travailler chez une tierce personne dans le domaine } \\
\text { agricole }\end{array}$ \\
\hline [trayzš $]$ (V.) & $\begin{array}{l}\text { - Se disputer / se prendre querelle. } \\
\text { - [t-taflat } \underline{?} \text { ?a-yatrayš- } u] \\
\text { - Les filles se disputent }\end{array}$ \\
\hline [htəš] (V.) & $\begin{array}{l}\text { - Couper l'herbe } \\
\text { - [ma nəmši nəhtəš l l-ba?ra] } \\
\text { - Je vais couper l'herbe pour la vache }\end{array}$ \\
\hline [hnna] (V.) & $\begin{array}{l}\text { - Aller / passer } \\
\text { - [hni \{and-əm l-ddār w Pull-əm yjiw] } \\
\text { - Passe chez eux à la maison et dis-leur de venir }\end{array}$ \\
\hline [rahhhot! (V.) & $\begin{array}{l}\text { - Courir } \\
\text { - [?a-ygəwwəz n-nhār Pa-yrahhhə̣t] } \\
\text { - Il passe la journée à courir. }\end{array}$ \\
\hline [Gむll] (V.) & $\begin{array}{l}\text { - Tourner / passer } \\
\text { - }[\text { sir gawd } w \text { Sđal } \text { Sla š-šmāl }] \\
\text { - Tu vas tout droit et tu tournes à gauche }\end{array}$ \\
\hline$[k a r f \partial t]$ (V.) & - Jeter / lancer quelque chose de manière aléatoire \\
\hline [wok $\underline{a} a]$ (V.) & $\begin{array}{l}\text { - Appuyer / s'appuyer. } \\
\text { - [wəkka Sla l- hayt] } \\
\text { - Il est adossé au mur }\end{array}$ \\
\hline [șbīh] (Adj.) & $\begin{array}{l}\text { - Beau / charmant. } \\
\text { - [ha tfila șbïha] } \\
\text { - Cette fillette est charmante. }\end{array}$ \\
\hline [mbəј̄̄̄?] (Adj.) & 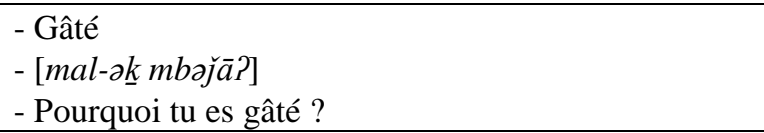 \\
\hline [sabā̃'] (Adv.) & 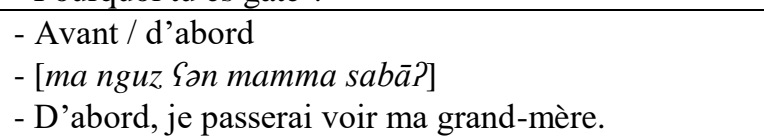 \\
\hline [šwiš] (Adv.) & $\begin{array}{l}\text { - Peu } \\
\text { - [tini šwiš }] ! \\
\text { - Donne-moi un peu ! }\end{array}$ \\
\hline
\end{tabular}




\section{Conclusion}

Comme nous l'avons bien signalé au début de ce papier, cette étude ne prétend pas présenter une description exhaustive du parler jebli des Ouled Boumalik, mais surtout de mettre en exergue les quelques traits saillants de ce parler préhilalien (spirants [t] [đ] [Đ] [k] / coup de glotte [?]...).

Il est à signaler que le parler en question présente aussi quelques termes manifestant le substrat amazighe (ex : mots commençant par -?a, désinence du masculin en amazighe). Il est bien nécessaire de procéder à des comparaisons bien poussées entre les différents parlers jeblis couvrant la région de Taounate, dans le cadre de cartes linguistiques.

\section{$* * *$ \\ BIBLIOGRAPHIE}

BRIGUI, Fouad. 2015. «Parlers arabes hilaliens du Nord-Ouest de Fès : Chraga, Hramssa et Ouled Aissa ». Dans : Actes de la journée d'études Linguistique de terrain : Description de faits et présentation de modèles. Fès, Publications du Laboratoire Langue, Littérature, Communication et Didactique, Imperssion SIPAMA, pp.33-46.

BRIGUI, Fouad \& GHILAN Fatima-Zahra. 2018. «De quelques éléments du vocabulaire de la vannerie chez les Bni Yazgha Un parler montagnard préhilalien méridional ». Dans : M. Mezzine, J. Vignet-Zunz \& F. Brigui (eds.). Jbala Peuplement, langue et ruralité. Rabat, Lawn, pp.97-104.

CHALFI, Rida. 2016. Étude de la variation langagière et des technolectes dans le parler jebli des Ouled Azam (province de Taounate). Thèse de doctorat, Kénitra, Faculté des lettres de Kénitra.

CHAFIK, Mohammed. 1989. Lamha an talata wa talatin qarnan min tarikhi al amazighiyin (Trad. Aperçu sur trente trois siècles d'histoire des Amazighs). Mohammedia, Alkalam.

IRAQUI-SINACEUR, Zakia. 1994. Dictionnaire Colin d'Arabe Dialectal marocain. Rabat, Editions Almanahil, Ministère des Affaires Culturelles.

IRAQUI-SINACEUR, Zakia. 1998. «Le dialecte de Tanger ». En : J. Aguadé, P. Cressier \& Vicente A. Dans : Peuplement et arabisation au Maghreb Occidental. Dialectologie et histoire. Madrid-Zaragoza, Casa de Velazquez, Universidad de Zaragoza, pp.121-130. 
LEVI-PROVENÇAL, Evariste. 1922. Textes arabes de L'Ouargha Dialecte des Jbala (Dialecte septentrional). Paris, Ernest Leroux.

MARÇAIS, Philippe. 1977. Esquisse grammaticale de l'arabe marocain, Paris, Maisonneuve.

MESSAOUDI, Leila. 1996. « Note sur l'affriquée [dj] dans le parler des Jbala (Nord du Maroc) ». EDNA 4, pp.167-175.

MESSAOUDI, Leila. 2003. Etudes sociolinguistiques. Rabat, Okad.

NATIVIDAD, Emma. 1998. «Le dialecte de Chefchaouen ». Dans : Aguadé J., Cressier P. \& Vicente A. Peuplement et arabisation au Maghreb Occidental. Dialectologie et histoire. Madrid-Zaragoza, Casa de Velázquez, Universidad de Zaragoza, pp.109-120.

VICENTE, Angeles. 1998. «Un dialecte de type montagnard au Maroc: le parler d'Anjra ». Dans : Aguadé J., Cressier P. \& Vicente A. Peuplement et arabisation au Maghreb Occidental. Dialectologie et Histoire, MadridZaragoza, Casa de Velázquez, Universidad de Zaragoza, pp.121-130.

VIGNET-ZUNZ, Jacques. 2015. «Le parler des Jbala : lexique». Dans : Actes $d u$ colloque international La situation des langues au Maroc : description linguistique et constitution de lexiques. Fès, Publications du Laboratoire Langue, Littérature, Communication et Didactique, Imperssion SIPAMA, pp.157-249.

ZIAMARI, Karima \& BARONTINI Alexandrine. 2008. «Quelques éléments de description d'un parler jebli (Ourtzagh, Maroc) ». EDNA 12, pp. 43-59. 